\title{
ß-GLUCAN-INDOMETHACIN COMBINATION PRODUCES NO LETHAL EFFECTS
}

\author{
Vaclav Vetvicka*, Jana Vetvickova
}

\author{
University of Louisville, Department of Pathology, Louisville, KY \\ e-mail:vetvickavaclav@netscape.net
}

Received: November 30, 2008; Accepted: January 17, 2009

Key words: Glucan/Indomethacin/Adverse effects/Phagocytosis/IL-6/Glucose

Background: The most important quality of $\beta$-glucans and the reason why so much attention has been devoted to them are their physiological effects. They are typical biological response modifiers with pronounced immunomodulating activity. However, some questions about possible side effects remain.

Aim: Several papers reported the lethal side effects of non-steroidal anti-inflammatory drugs in glucan-treated mice, probably due to the peritonitis by enteric bacteria. However, these results were never independently confirmed. The purpose of this study is to evaluate these claims using several different types of glucans.

Methods: Effects of combined treatment with four different types of glucans and indomethacin were measured by evaluation of phagocytosis of HEMA particles by peripheral blood leukocytes and production of IL-6 in mouse serum. In addition, the level of blood glucose, colon length and survival after 30 days of treatment was measured.

Results: Our finding showed that simultaneous treatment with glucan and indomethacin caused a small decrease of phagocytic activity and IL-2 production. Two other tested parameters-blood glucose levels and colon length-that had been found to be significantly affected by this treatment, were virtually unchanged. In the final, yet most important part of the study, we found absolutely no mortality, regardless of the type of glucan or the routes of glucan administration.

Conclusion: No adverse negative effects due to simultaneous treatment with glucan and non-steroidal anti-inflammatory drug dose was found, despite testing two different routes of glucan administration and four different types of glucan.

\section{INTRODUCTION}

Indomethacin is a non-steroidal anti-inflammatory drug suppressing prostaglandin synthesis via inhibition of cyclooxygenase. Despite well known positive effects, adverse side effects including mucosal damage on the gastrointestinal tract exist ${ }^{1,2}$.

$\beta$-Glucans are natural immunomodulators gaining significant interest from both the scientific community and the general public. These polysaccharides are noncellulosic polymers of $\beta$-glucose, with glycosidic bonds in position $\beta(1 \rightarrow 3)$ and with certain portion of $\beta(1 \rightarrow 6)$ branched glucose molecules. They are isolated mainly from various fungi, but they are also present in other sources, such as cereals, bacteria or seaweed. These compounds, isolated from different sources that otherwise caused similar or nearly identical immune reactions in macroorganisms, can differ in their primary, secondary or tertiary structures or their solubility in water or alkalies (for review see ${ }^{3}$ ).

The most important quality of $\beta$-glucans and the reason why so much attention has been devoted to them are physiological effects that they reveal. They are typical biological response modifiers with pronounced immunomodulating activity. In addition, over 6,000 scientific papers have been devoted to biological actions of glucans.

Thus far, the strong immunostimulating properties of glucan are the best studied effects. The best known effects of $\beta$-glucans consist of the augmentation of phagocytosis of professional phagocytes - granulocytes, monocytes, macrophages and dendritic cells. In this regard, macrophages, considered to be the basic effector cells in host defense against bacteria, viruses, parasites and tumor cells, play the most important role ${ }^{4}$.

Decade of studies of the Ross group help to elucidate the mechanisms of the glucan action. Our research has shown that CR3 serves as a major receptor for $\beta$-glucans with human ${ }^{5}$ or mouse ${ }^{4}$ leukocytes and is probably responsible for reported functions of $\beta$-glucans. $\beta$-Glucan specifically targets macrophages, neutrophils, and NK cells to tumors that are opsonized with antibodies and complement. Thus, $\beta$-glucan has the same specificity as the tumor-opsonizing antibodies. Studies in mice have shown the therapeutic value of small soluble $\beta$-glucans (5-20 kD) that can bind to CR3 monogamously and prime the receptor for subsequent cytotoxic activation only if membrane CR 3 are subsequently clustered by contact with the clustered iC $3 \mathrm{~b}$ coating a tumor cell. High m.w. soluble $\beta$-glucans such as lentinan (> $500 \mathrm{kD}$ ) that have been used for patient therapy are large enough to crosslink and cluster membrane CR3, triggering respiratory bursts, degranulation, and cytokine release in the absence of tumor target cells ${ }^{6,7}$. Several studies have shown the safety of soluble $\beta$-glucans and the absence of side effects. It should further be noted that small soluble polysaccharides are rarely antigenic unless coupled to proteins.

Our knowledge of possible negative effects of various $\beta$-glucans is limited. Particulate $\beta$-glucan applied parenterally was reported to cause granuloma formation, microembolization, local inflammation and pain ${ }^{8,9}$. Inhalation 
of intact cells or cellular detritus of fungi or yeasts-ingredients of home dust ${ }^{10}$ or different agricultural and industrial dusts ${ }^{11}$-induces the so called syndrome of toxic organic dust which is characterized by lung reactions that include pneumonia, cough, chronic bronchitis ${ }^{12}$, rhinitis, headache and irritation of eyes and throat. $\beta$-Glucans were reported as important causal factors of these complaints ${ }^{13}$ which, through activation of macrophages, monocytes and leukocytes, causes increased secretion of inflammatory components (e.g., TNF- $\alpha$ and interleukin-8 (IL$8)$ ). $\beta$-Glucans have also been suggested to be important agents in the inflammatory reactions seen in the so called Sick Building Syndrome ${ }^{14}$.

A major problem was evidenced when glucan was applied simultaneously with indomethacin. Several papers reported the lethal side effects of indomethacin in glucan-treated mice ${ }^{15-18}$. These authors found that in glucan-treated mice, non-steroidal anti-inflammatory drug caused a gastrointestinal damage resulting in peritonitis by enteric bacteria and subsequent death. The findings that lincomycin protected the animals from septic shock in these glucan-indomethacin model further supported the theory of bacterial infection. However, these results were never independently confirmed. The purpose of this study is to evaluate these claims using several different types of glucans.

\section{MATERIALS AND METHODS}

\section{Animals}

Female, 6 to 10 week old BALB/c mice were purchased from the Jackson Laboratory (Bar Harbor, ME). All animal work was done according to the University of Louisville IACUC protocol. Animals were sacrificed by $\mathrm{CO}_{2}$ asphyxiation.

\section{Material}

RPMI 1640 medium, sodium citrate, Wright stain, and indomethacin were obtained from Sigma Chemical Co. (St. Louis, MO), fetal calf serum (FCS) was from Hyclone Laboratories (Logan, UT).

\section{$\beta-1,3$ glucans}

The glucans used in this study were purchased from the following companies: NOW BETA glucan from NOW FOODS (Bloomingdale, IL), Epicor from from Embria Health Sciences (Ankeny, IA), Glucan \#300 from Transfer Point (Columbia, SC), and Glucagel $\mathrm{T}$ from GraceLinc (Christchurch, New Zealand).

\section{Glucan treatment}

Individual glucans were applied either intraperitoneally or orally. The samples were collected at different intervals after either ip. injections (100 $\mu \mathrm{g}$ of glucan/ mouse) or after feeding with glucan-containing diet. All diets (Laboratory Rodent Diet 5001 enhanced with various doses of glucan) were formulated and prepared by Purina (Richmond, IN). Diet ingredients for all groups were identical except for the proportion of glucan.

\section{Phagocytosis}

The technique employing phagocytosis of synthetic polymeric microspheres was described earlier ${ }^{19}$. Briefly: peritoneal cells were incubated with $0.05 \mathrm{ml}$ of 2-hydroxyethyl methacrylate particles (HEMA; $5 \times 10^{8} / \mathrm{ml}$ ). The test tubes were incubated at $37^{\circ} \mathrm{C}$ for $60 \mathrm{~min}$., with intermittent shaking. Smears were stained with Wright stain. The cells with three or more HEMA particles were considered positive. The same smears were also used for evaluation of cell types.

\section{Cytokine assay}

$\mathrm{BALB} / \mathrm{c}$ mice were intraperitoneally injected with $100 \mu \mathrm{g}$ of glucan and fed with indomethacin. Control mice obtained PBS only. After 24 hrs, the mice were sacrificed and blood was collected in Eppendorf tubes. Subsequently, the serum was prepared, collected and stored at $-80{ }^{\circ} \mathrm{C}$ for no more than 1 week. Alternatively, glucans $(100 \mu \mathrm{g} / \mathrm{mouse}$ in PBS) were administered ip. 3 times on days $-5,-3$, and -1 . Indomethacin $(5 \mathrm{mg} / \mathrm{kg}$ ) was administered p.o. from day 0 to 5 .

The levels of IL-6 in serum samples were evaluated using a commercial kit OptEIA Mouse IL-6 set (Pharmingen, San Diego, CA, USA) according to the manufacturer's instructions. The optical density was determined using a STL ELISA reader (Tecan U.S., Research Triangle Park, NC) at $450 \mathrm{~nm}$ with a correction at $570 \mathrm{~nm}$. Data shown were calculated from the standard curve prepared by the automated data reduction using linear regression analysis. A standard curve was run with each assay.

\section{Gastrointestinal length}

The entire colon, from the coecum to the bottom of the rectum, was removed and colon length was measured as an indirect marker of inflammation ${ }^{18}$.

\section{Glucose}

Mice were deprived of food for $24 \mathrm{hr}$ and sacrificed. Serum was collected via the retro-orbital sinus and stored at $-80{ }^{\circ} \mathrm{C}$ for less ten a week. Biochemical analyses were performed by Antech Diagnostics (Indianapolis, IN).

\section{Survival experiments}

In Group A, glucan was injected at days $-5,-3$ and -1 , indomethacin in delivered water from day 1 to day 30 . In Group B, same doses of glucan and or indomethacine were delivered orally from day 1 to day 30 . The mice were observed daily and survival was counted. The experiment was stopped after 30 days.

\section{RESULTS}

The number of individual glucans is almost as great as the number of sources used for their isolation. The rationale for this combination of glucan samples was not only their commercial availability and success but, most importantly, we tried to include both soluble and insoluble 
glucans and also glucans from different sources, including yeast, mushrooms and cereals.

Glucans are generally considered to be potent stimulators of cellular immunity, with macrophages and neutrophils being the most important targets. Not surprisingly, we started our evaluation of glucan activities by phagocytosis. We used the synthetic polymeric microspheres, HEMA, since their use, dose, and timing are already well established in glucan studies. ${ }^{20}$ Results summarized in Fig. 1 show significant effects of glucans \#300 and Now on encapsulation of synthetic particles by peripheral blood neutrophils. At the same time, indomethacine significantly lowered the phagocytic activity. However, three glucans- \#300, Now and glucage- returned this suppression to almost normal values.

Next, we measured the level of IL-6 in serum of mice treated with glucan and indomethacin for $24 \mathrm{hr}$. Results summarized in Fig. 2 show that all glucans stimulated the IL-2 secretion. The simultaneous addition of indomethacin resulted in small and, in most cases, insignificant suppression of IL-6 secretion. The suppression was significant, only in thecase of Now glucan. Longer exposure to glucans resulted in higher IL-6 secretion (Fig. 3) and longer exposure to indomethacin resulted in lower IL-6 production. Glucans \#300 and Epicor managed to buffer the indomethacin-induced suppression.

Serum glucose concentrations of glucan-treated mice was compared with those treated with glucan- and indomethacin. On day 5, mice were sacrificed and sera were collected. As shown in Fig. 4, the serum glucose levels were not significantly affected by either glucan alone or by indomethacine alone.

Intestinal injury is common in indomethacin-treated subjects and colon shortening is considered to be a characteristic sign of the inflammatory bowel disease. Therefore, we measured effect of glucan and/or indomethacin on the colon length. Glucans $(100 \mu \mathrm{g} /$ mouse in PBS) were administered ip. 3 times on days $-5,-3$, and -1 , indomethacin $(5 \mathrm{mg} / \mathrm{kg})$ was administered p.o. from day 0-to-5. Colons were obtained from each group and measured on day 5. Glucan alone had no effects on colon length. Similarly, indomethacin alone caused no colon shortening. When combined, no shortening of the colon was observed. Conversely, simultaneous treatment with Epicor and indomethacin resulted in significant increase of colon length.

Finally, we addressed the most important question does the simultaneous treatment of mice with glucan and indomethacin really affect the lethality? To examine the effect of this combination, we measured the mortility of animals after 30 days of treatment. Results given in Table 1 showed that, regardless of the administration of the glucan, absolutely no changes in mice survival were found.

\section{DISCUSSION}

$\beta$-Glucans belong to a group of natural, physiologically active compounds, generally called biological response modifiers.
Table 1. Effect of combination treatment of glucan and indomethacin on mouse survival.

\begin{tabular}{|l|c|}
\hline \multicolumn{2}{|c|}{ Group A } \\
\hline after 30 days \\
\hline \#300 + IND & $9 / 9$ \\
\hline Epicor + IND & $9 / 9$ \\
\hline Glucagel + IND & $8 / 8$ \\
\hline Now + IND & $9 / 9$ \\
\hline \multicolumn{2}{|c|}{ Group B } \\
\hline \#300 + IND & \multicolumn{1}{|c|}{$8 / 8$} \\
\hline Epicor + IND & $9 / 7$ \\
\hline Glucagel + IND & $9 / 9$ \\
\hline Now + IND & \multicolumn{1}{|c|}{$9 / 9$} \\
\hline Group A & $\begin{array}{l}\text { Glucan injected at days }-5,-3 \\
\text { and }-1, \text { indomethacin in water } \\
\text { from day 1 to 30 }\end{array}$ \\
\hline Group B & $\begin{array}{l}\text { Glucan in food, indomethacin } \\
\text { in water from day } 1 \text { to } 30\end{array}$ \\
\hline
\end{tabular}

The best known effects of $\beta$-glucans consist of the augmentation of phagocytosis of professional phagocytes, granulocytes, monocytes, macrophages and dendritic cells. Activation of macrophages and other professional phagocytes represents an element of more complicated processes, when mediator molecules secreted by them (such as IL-1, IL-9, or TNF- $\alpha$ ) initiate inflammation reactions. Additional studies established that glucans also stimulate the humoral branch of immune reactions ${ }^{21}$. In addition, glucans were repeatedly shown to overcome the immunosuppression, caused by either irradiation or chemotherapy ${ }^{21,22}$.

The rationale for the choice of glucans parallels what was stated in our previous paper ${ }^{20}$. We chose four glucans that are widely sold and available in the US, Europe and the Far East, representing grain-, mushroom- and yeastderived glucans in partly soluble and insoluble form. Briefly, \#300 is insoluble yeast-derived glucan; Glucagel is barley-derived glucan; NOW is a mixture of both insoluble glucans from yeast and soluble glucans from mushrooms; and Epicor is a partly soluble yeast-derived glucan.

Despite the fact that most reports found no significant side effects connected with glucan treatment (for review $\mathrm{see}^{3}$ ), including long-term use ${ }^{23}$. One potential question remains - are there lethal side effects when glucan is combined with indomethacin? Several reports described that $\beta$-glucan-induced inflammatory processes could competitively interact with simultaneously administered nonsteroidal antiinflammatory drugs. The lethal toxicity due to septic shock, elicited by a sequential administration of $\beta$-glucan and a nonsteroidal anti-inflammatory drug indomethacin, was described in mice ${ }^{15,16}$. In addition, these effects can be reversed by antibiotics ${ }^{17,18}$. Unfortunately, all these reports are from one laboratory and despite the 


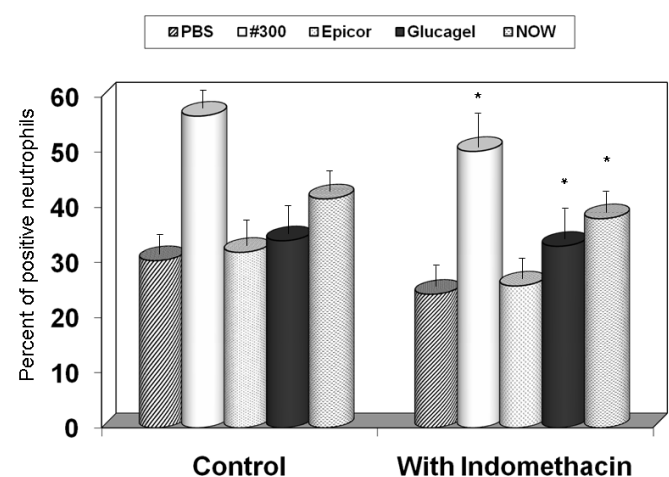

Fig. 1. Role of glucans on phagocytic activity of blood neutrophils. Glucan (100 $\mu \mathrm{g} /$ mouse in PBS) was administered ip. 3 times on days $-5,-3$, and -1 . Indomethacin $(5 \mathrm{mg} / \mathrm{kg})$ was administered p.o. from day 0 to 5 . *Represents significant differences between control (indomethacin) and samples (indomethacin and glucan) at $\mathrm{P} \leq 0.05$ level.

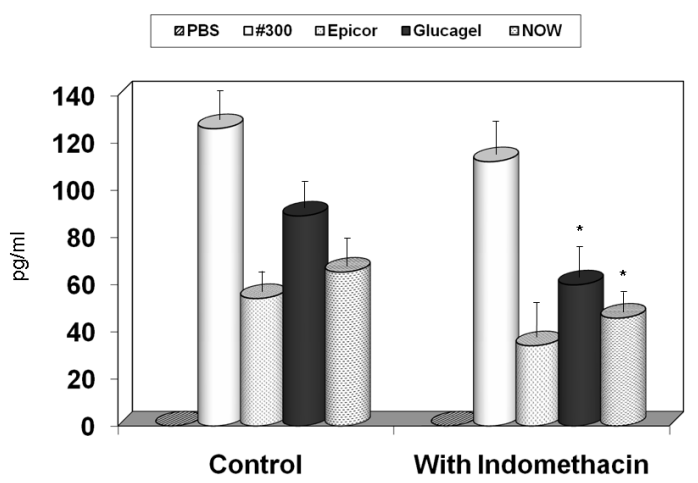

Fig. 3. Role of glucans and indomethacin on the level of IL-6 in serum 5 days after application of tested substaneces. Glucan ( $100 \mu \mathrm{g} /$ mouse in PBS) was administered ip. 3 times on days $-5,-3$, and -1 . Indomethacin $(5 \mathrm{mg} / \mathrm{kg})$ was administered p.o. from day 0 to 5 . *Represents significant differences between group without indomethacin and and group with indomethacin at $\mathrm{P} \leq 0.05$ level.

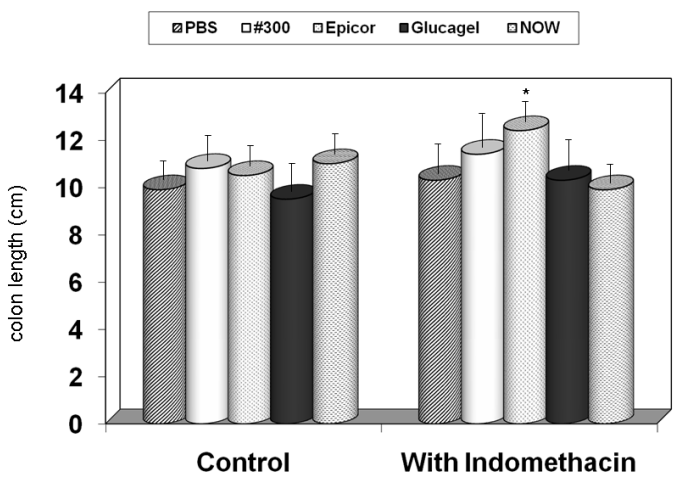

Fig. 5. Glucan ( $100 \mu \mathrm{g} /$ mouse in PBS $)$ was administered ip. 3 times on days $-5,-3$, and -1 . Indomethacin $(5 \mathrm{mg} / \mathrm{kg})$ was administered p.o. from day 0 to 5. Colons were obtained from each group and measured on day 5 . Number of mice used in each group was 3.

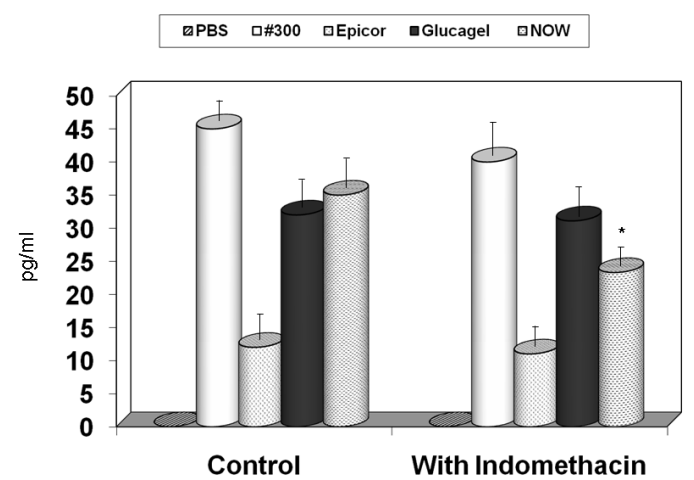

Fig. 2. Role of glucans and indomethacin on the level of IL-6 in serum $24 \mathrm{hrs}$ after application (glucan ip, indomethacin orally). *Represents significant differences between group without indomethacin and and group with indomethacin at $\mathrm{P} \leq 0.05$ level.

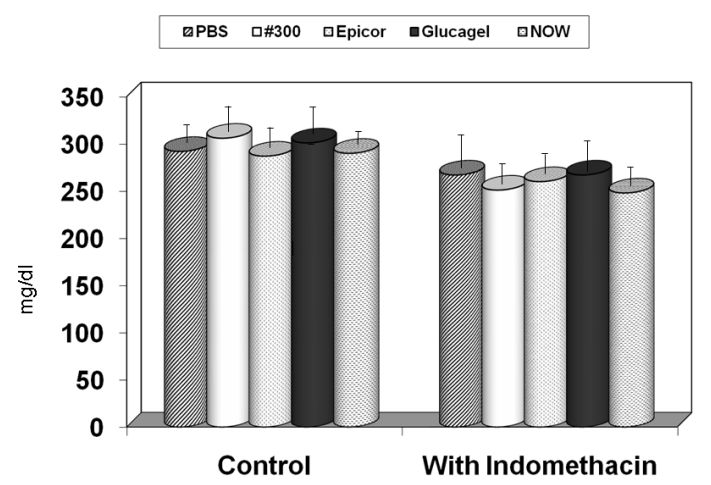

Fig. 4. Role of glucans and indomethacin on the level of glucose in serum 5 days after application of tested substaneces. Glucan $(100 \mu \mathrm{g} /$ mouse in PBS) was administered ip. 3 times on days $-5,-3$, and -1 . Indomethacin $(5 \mathrm{mg} / \mathrm{kg})$ was administered p.o. from day 0 to 5 .

fact that these effects were repeatedly described for more than 10 years, they were never independently confirmed. The aim of this report was, therefore, to either confirm or reject the current hypothesis about the negative association of glucan and non-steroidal anti-inflammatory drugs.

We have used the same conditions as described in ${ }^{18}$. In order to be sure we evaluate all possibilities, we included the experimental group where glucan was also administered orally. In addition, as individual glucan often differ in their biological properties, we wanted to establish that our findings had general meaning. Therefore, we used four different types of glucan, differing in source, solubility and activity,

Our finding showed that simultaneous treatment with glucan and indomethacin caused small decrease of phagocytic activity and IL-2 production. Two other tested parameters-blood glucose levels and colon length-both found to be significantly affected by this treatment ${ }^{18}$, were 
virtually unchanged. The reason why Epicor increased the colon length is unknown at this time.

In the final, yet most important part of the study, we found absolutely no mortality, regardless the type of glucan or the route of glucan administration. One explanation of the differences between our findings and Ohno's group might be that the original papers used the only type of glucan with particularly peculiar biological properties that cannot be found in any other glucan.

There is evidence that $\beta$-glucan plays a considerable role in increased production of nitric oxide, one of the most effective reactive nitrogen species, by inducible nitric oxide synthase (iNOS) in macrophages ${ }^{24,25}$ from L-arginine. Various types of nitric oxide synthase (NOS) are known (besides iNOS also a neuronal and epithelial ones). Function of formed nitric oxide is double-edged. It induces a cytotoxic effect upon tumor cells ${ }^{26}$ and shows distinct impact on many pathogens ${ }^{27}$. On the other hand, it can damage tissues and DNA ${ }^{28}$ and its high concentrations can play role in septic shock. The activator's sustained action induces expression of iNOS and increased formation of nitric oxide (NO) results in vasodilatation of veins. The vasodilatation brings about an intense drop of venous resistance and blood pressure ${ }^{29}$. Other side effects imputed to $\beta$-glucan-induced nitric oxide production are bioaerosol-induced respiratory symptoms seen in both occupational and residential environments ${ }^{30}$. Generally, the dual role of NO as a protective or toxic molecule is due to several factors such as the isoform of NOS involved, concentration of NO and the type of cells in which it is synthesized, the availability of L-arginine, formation of cGMP by soluble guanylate cyclase, and the overall extra and intracellular environment in which NO is produced ${ }^{31}$.

It is important to note that there are some limitations of this study, as some of the observed markers are not full markers of sepsis (e.g. glycemia). Similarly, colon length is not by some considered to be an adequate marker of inflammation. However, the most important result of our study was the finding of absolutely no lethal effects of the glucan-indomethacin combination, making the relevance of the supporting markers significantly less relevant.

There are two problems with natural $\beta$-glucans that make them undesirable as drugs. First, there is considerable variation in the structure of $\beta$-glucans isolated from the same strain of fungi. The second problem in the development of a drug from a natural product is that it is difficult to make it proprietary and protect the investment required for development. However, based on results of this study, the adverse negative effects of the simultaneous treatment with glucan and non-steroidal anti-inflammatory drugs does not seem to be another problem.

\section{REFERENCE}

1. Erdeve O, Sarici SU, Sari E, Gok F. (2008) Oral-ibuprofen-induced acute renal failure in a preterm infact. Pediatr. Nephrol., 23, 15661567.

2. Sing S, Khajuria A, Taneja SC, Khajuria RK, Singh J, Jihri RK, Qazi GN. (2008) The gastric ulcer protective effect of boswell- ic acids, a leukotriene inhibitor from Boswellia serrata, in rats. Phytomedicine 15, 408-415.

3. Novak M, Vetvicka V. (2008) Beta-glucans, history, and the present: Immunomodulatory aspects and mechanisms of action, J. Immunotoxicol., 5, 47-57.

4. Vetvicka V, Thornton BP, Ross GD. (1996) Soluble $\beta$-glucan polysaccharide binding to the lectin site of neutrophil or natural killer cell complement receptor type 3 (CD11b/CD18) generates a primed state of the receptor capable of mediating cytotoxicity of iC3b-opsonized target cells.J. Clin. Invest., 98, 50-61.

5. Czop JK, Austen KF. (1985) Properties of glycans that activate the human alternative complement pathway and interact with the human monocyte $\beta$-glucan receptor. J. Immunol., 135, 3388-3393.

6. Janusz MJ, Austen KF, Czop JK. (1989) Isolation of a yeast heptaglucoside that inhibits monocyte phagocytosis of zymosan particles. J. Immunol., 142, 959-965.

7. Jamois F, Ferrieres V, Guegan J-P, Yvin J-C, Plusquellec D, Vetvicka V. (2005) Glucan-like synthetic oligosaccharides - Iterative synthesis of linear oligo- $\{\beta\}-(1,3)$-glucans and immunostimulatory effects. Glycobiology 15, 393-407.

8. DiLuzio NR, Williams DL, McNamee RB, Edwards BF, Kitahame A. (1979) Comparative tumor-inhibitory and anti-bacterial activity of soluble and particulate glucan. Internat, J. Cancer 24, 773-779.

9. Zekovic DB, Kwiatkowski S, Vrvic MM, Jakovljevic D, Moran CA. (2005) Natural and modified (1->3)-beta-D-glucans in health promotion and disease alleviation. Crit. Rev. Biotechnol., 25, 205-230.

10. Rylander R, Thorn J, Attefors R. (1999) Airways inflammation among workers in a paper industry. Eur. Respir. J., 13, 1151-1158.

11. Sato M, Sano H. (2003) Direct binding to Toll-like receptor 2 to zymosan, and zymosan-induced NF-kappa B activation and TNFalpha secretion are down-regulated by lung collection surfactant protein. J. Immunol., 171, 417-425.

12. Alwis KU, Mandryk J, Hocking AD. (1999) Exposure to biohazards in wood dust: bacteria, fungi, endotoxins, and (1->3)-beta-Dglucans. Appl. Occupat. Environment. Hyg., 14, 598-608.

13. Alwis KU, Milton DK. (2006) Recombinant factor C assay for measuring endotoxin in house dust: comparison with LAL, and (1 -> 3)-beta-D-glucans. Am. J. Ind. Med., 49, 296-300.

14. Ljungman AG, Leanderson P, Tagesson C. (1998) (1->3)- $\beta-D-$ glucan stimulates nitric oxide generation and cytokine mRNA expression in macrophages. Environ. Toxicol. Pharmacol., 5, 273-281.

15. Yoshiba S, Ohno N, Miura ST, Adachi Y, Yadomae T. (2001) Immunotoxicity of soluble $\beta$-glucans induced by indomethacin treatment. FEMS Immunol. Med. Microbiol., 21, 171-179.

16. Takahasmi H, Ohno N, Adachi Y, Yadomae T. (2001) Association of immunological disorders in lethal side effects of NSAIDs on $\beta$-glucan-administered mice. FEMS Immunol. Med. Microbiol., 31, 1-14.

17. Nameda S, Miura NN, Adachi Y, Ohno N. (2007) Antibiotics protect against septic shock in mice administered $\beta$-glucan and indomethacin. Microbiol. Immunol., 51, 851-859.

18. Nameda S, Miura NN, Adachi Y, Ohno N. (2007) Lincomycin protects mice from septic shock in $\beta$-glucan-indomethacin model. Biol. Pharm. Bull., 30, 2312-2316.

19. Vetvicka V, Fornusek L, Kopecek J, Kaminkova J, Kasparek L, Vranova M. (1982) Phagocytosis of human blood leukocytes: A simple micromethod. Immunol. Lett. 5, 97-100.

20. Vetvicka V, Vetvickova J. (2007) An evaluation of the immunological activities if commercially available $\beta$-1,3-glucans. J. Amer. Nutr. Assoc., 10, 25-31.

21. Vetvicka V, Dvorak B, Vetvickova J, Richter J, Krizan J, Sima P, Yvin JC. (2006) Orally administered marine (1->3)-beta-D-glucan Phycarine stimulates both humoral and cellular immunity. Int. J. Biol. Macromol., 40, 291-298.

22. Patchen ML, MacVittie TJ. (1982) Use of glucan to enhance hemopoietic recovery after exposure to cobalt-60 irradiation, Adv. Exp. Med. Biol., 155, 267-272.

23. Babicek K, Cechova I, Simon RR, Harwood M, Cox DJ. (2007) Toxicological assessment of a particulate yeast $(1,3 / 1,6)-\beta$-D-glucan in rats, Food Chem. Toxicol., 45, 1719-1730. 
24. Ohno N, Egawa Y, Hashimoto T, Adachi Y, Yadomae T. (1996) Effect of beta-glucans on the nitric oxide synthesis by peritonea macrophage in mice. Biol. Pharm. Bull., 19, 608-612.

25. Hashimoto T, Ohno N, Adachi Y, Yadomae T. (1997) Enhanced production of inducible nitric oxide synthase by $\beta$-glucans in mice. FEMS Immunol. Med. Microbiol., 19, 134-135.

26. Hibbs JB, Taintor RR, Vavrin Z. (1984) Iron depletion: possible cause of tumor cell cytotoxicity induced by activated macrophages. Biochem. Biophys. Res. Commun., 123, 716-723.

27. James S, Glaven J. (1989) Macrophage cytotoxicity against schistosomula of Schistosoma mansoni involves arginine-dependent production of reactive nitrogen intermediates. J. Immunol., 143, 4208-4212.
28. Billiar TR. (1995) Nitric oxide novel biology with clinical relevance. Ann. Surg., 221, 339-349.

29. Vincent JL, Zhang H, Szabo C, Preiser JC. (2000) Effects of nitric oxide in septic shock. Am. J. Respir. Crit. Care Med., 161, 17811785.

30. Beijer L, Thorn J, Rylander R. (2002) Effects after inhalation of (1->3)-beta-D-glucan and relation to mould exposure in the home. Mediators Inflam., 11, 149-153.

31. Thippeswamy T, McKay JS, Quinn JP, Morris R. (2006) Nitric oxide, a biological double-faced janus - is this good or bad? Histol. Histopathol., 21, 445-448. 